\title{
Generalization of the Cover Pebbling Number for Networks
}

\author{
Zheng-Jiang Xia* and Zhen-Mu Hong \\ School of Finance, Anhui University of Finance \& Economics, Bengbu, China
}

Pebbling can be viewed as a model of resource transportation for networks. We use a graph to denote the network. A pebbling move on a graph consists of the removal of two pebbles from a vertex and the placement of one pebble on an adjacent vertex. The $t$-pebbling number of a graph $G$ is the minimum number of pebbles so that we can move $t$ pebbles on each vertex of $G$ regardless of the original distribution of pebbles. Let $\omega$ be a positive function on $V(G)$; the $\omega$-cover pebbling number of a graph $G$ is the minimum number of pebbles so that we can reach a distribution with at least $\omega(v)$ pebbles on $v$ for all $v \in V(G)$. In this paper, we give the $\omega$-cover pebbling number of trees for nonnegative function $\omega$, which generalized the $t$-pebbling number and the traditional weighted cover pebbling number of trees.

Keywords: network, tree, path partition, pebbling, cover pebbling

\section{OPEN ACCESS}

Edited by:

Jia-Bao Liu,

Anhui Jianzhu University, China

Reviewed by:

Fu-Tao Hu,

Anhui University, China

Zhi Qiao,

Sichuan Normal University, China Xiang-jun Li,

Yangtze University, China

*Correspondence:

Zheng-Jiang Xia 120150025@aufe.edu.cn

Specialty section

This article was submitted to Mathematical Physics,

a section of the journal

Frontiers in Physics

Received: 12 April 2020

Accepted: 04 May 2020

Published: 16 June 2020

Citation:

Xia Z-J and Hong Z-M (2020) Generalization of the Cover Pebbling

Number for Networks.

Front. Phys. 8:197.

doi: 10.3389/fphy.2020.00197
Mathematics Subject Classification: 05C99, 05C72, 05 C85.

\section{INTRODUCTION}

Pebbling in graphs was introduced by Chung [1]. It can also be viewed as a model of resource transportation for networks. Let $G$ be a simple connected graph; we use $V(G)$ and $E(G)$ to denote the vertex set and edge set of $G$, respectively. $d(u, v)$ is the distance of $u$ and $v$, and we write $u \sim v$ if they are adjacent. $N(v)=\{u \mid u \sim v\}$ is the neighbor of $v$, and $d(v)=|N(v)|$ is the degree of $v$. Let $H$ be a subgraph of $G$; we use $d_{H}(v)$ to denote the degree of $v$ in $H$.

A pebble distribution $D$ on $G$ is a function $D: V(G) \rightarrow N$ ( $N$ is the set of nonnegative integers), where $D(v)$ is the number of pebbles on $v,|D|=\sum_{v \in V(D)} D(v)$ is the size of $D$.

A pebbling move consists of the removal of two pebbles from a vertex and the placement of one pebble on an adjacent vertex. Let $D$ and $D^{\prime}$ be two pebble distributions of $G$. If so, we say that $D$ contains $D^{\prime}$ if $D(v) \geq D^{\prime}(v)$ for all $v \in V(G)$, and $D^{\prime}$ is reachable from $D$ if there is some sequence (probably empty) of pebbling moves (a pebbling sequence in short) starting from $D$ and resulting in a distribution, which contains $D^{\prime}$. For a graph $G$ and a vertex $v$, we call $v$ a root (or target vertex) if the goal is to place pebbles on $v$. If $t$ pebbles can be moved to $v$ from $D$ by a sequence of pebbling moves, then we say that $D$ is $t$-fold $v$-solvable, and $v$ is $t$-reachable from $D$. If $D$ is $t$-fold $v$-solvable for every vertex $v$, we say that $D$ is $t$-solvable.

The $t$-pebbling number of a graph $G$, denoted by $f_{t}(G)$, is the smallest number $m$ such that every distribution with size $m$ is $t$-solvable. While $t=1$, we use $f(G)$ instead of $f_{1}(G)$, which is called the pebbling number of $G$.

For any two graphs $G$ and $H$, we define the Cartesian product $G \times H$ to be the graph with the vertex set $V(G \times H)$ and edge set the union of $\{((a, v),(b, v)) \mid(a, b) \in E(G), v \in E(H)\}$ and $\{((u, x),(u, y)) \mid u \in V(G)$, and $(x, y) \in E(H)\}$.

To determine the pebbling number of a general graph $G$ is difficult. The problem of whether a distribution is $v$-solvable for some $v \in V(G)$ was shown to be NP-complete [2,3]. The problem of 
deciding whether the pebbling number of a graph $G$ is less than $k$ was shown to be $\Pi_{2}^{P}$-complete [3]. The pebbling numbers of trees [4], cycles [5], hypercubes [1], and so on are determined. A conjecture called Graham's Conjecture is given by Chung [1].

Conjecture 1.1. (Graham's Conjecture) Let $G$ and $H$ be two connected graphs; the pebbling number of the Cartesian product of $G$ and $H$ satisfies:

$$
f(G \times H) \leq f(G) f(H)
$$

There are many results about Graham's Conjecture [6-10], while this conjecture is still open.

Definition 1.2. Let $\omega$ be a nonnegative function on $V(G)$ and $D$ a distribution on $V(G)$. We say $D$ is $\omega$-solvable (or $D$ solves $\omega)$ if we can reach a distribution $D^{*}$ from $D$, by a sequence of pebbling moves, so that $D^{*}(v) \geq \omega(v)$ for all $v \in V(G)$. The $\omega$ cover pebbling number of $G$, denoted by $\gamma_{\omega}(G)$, is the minimum number $m$ so that every distribution $D$ with size $m$ is $\omega$-solvable.

Definition 1.3. Let $\omega$ be a positive function on $V(G)$; define

$$
s_{\omega}(v)=\sum_{u \in V(G)} \omega(u) 2^{d(u, v)},
$$

and

$$
s_{\omega}(G)=\max _{v \in V(G)} s_{\omega}(v) .
$$

The $\omega$-cover pebbling number of a graph $G$ has been determined for positive $\omega$ by [11].

Theorem 1.4. ([11]) Let $\omega$ be a positive weight function on $V(G)$; the $\omega$-cover pebbling number of $G$ is

$$
\gamma_{\omega}(G)=s_{\omega}(G)
$$

From Theorem 1.4, we can get

Theorem 1.5. ([11]) Let $\omega_{1}$ be a positive function on $G$ and $\omega_{2}$ be a positive function on $H$. The function $\omega$ on $G \times H$ is given by $\omega((g, h))=\omega_{1}(g) \omega_{2}(h)$, where $g \in V(G)$ and $h \in V(H)$, then $\gamma_{\omega}(G \times H)=\gamma_{\omega_{1}}(G) \gamma_{\omega_{2}}(H)$.

We first generalize the definition of $s_{\omega}(T)$ while $\omega$ is a nonnegative function on a tree $T$. We will give the definition of path partition in the next section.

Definition 1.6. Given a tree $T$ and a nonnegative function $\omega$ for each vertex $v \in V(T)$, and let $T_{\omega}(v)$ be the minimum subtree of $T$ containing $v$ and $W:=\{u: \omega(u)>0\}$. We give each edge in $T \backslash E\left(T_{\omega}(v)\right)$ a direction toward $T_{\omega}(v)$ to get a directed graph, which is denoted by $\vec{T} \backslash E\left(T_{\omega}(v)\right)$, and $\left(a_{1}, \ldots, a_{n}\right)$ is the size of the maximum path partition of $\vec{T} \backslash E\left(T_{\omega}(v)\right)$. We define

$$
s_{\omega}(v)=\sum_{u \in W} \omega(u) 2^{d(u, v)}+\sum_{i=1}^{n} 2^{a_{i}}-n .
$$

and

$$
s_{\omega}(T)=\max _{v \in V(T)} s_{\omega}(v) .
$$

Note that while $\omega$ is positive, then the two definitions of $s_{\omega}(T)$ are the same. Definition 1.6 is thus a generalization of Definition 1.3. In this paper, we generalize Theorem 1.4 while $T$ is a tree and $\omega$ is nonnegative. Thus, our main result is as follows

Theorem 1.7. Let $T$ be a tree with a nonnegative weight function $\omega$ on $V(T)$. The $\omega$-cover pebbling number of $T$ is

$$
\gamma_{\omega}(T)=s_{\omega}(T)
$$

Theorem 1.8. Let $T$ be a tree with a nonnegative weight function $\omega$ on $V(T)$. If $|W|=1$, then Theorem 1.7 is equivalent to Theorem 2.2.

Proof. If $|W|=1$, assume that $\omega(v)=t$, and $\omega(u)=0$ for $u \neq v$. We will show that $f_{t}(T, v)=s_{\omega}(T)$.

Assume the size of a maximum path partition of $\vec{T}_{v}$ is $\left(a_{0}, a_{1}, \ldots, a_{n}\right)$, and $d\left(v, v_{0}\right)=a_{0}, P_{0}$ is the longest directed path from $v_{0}$ to $v$. Then $\left(a_{1}, \ldots, a_{n}\right)$ must be the size of a maximum path partition in $\vec{T}_{v} \backslash P_{0}$. So $f_{t}(T, v)=s_{\omega}\left(v_{0}\right) \leq s_{\omega}(T)$.

Assume $s_{\omega}(T)=s_{\omega}\left(v_{1}\right)$, and $d\left(v_{1}, v\right)=a_{0}$. Let $P_{0}$ be the path connected $v_{1}$ and $v$, then $T_{\omega}\left(v_{1}\right)=P_{0}$; assume $\left(a_{1}, \ldots, a_{n}\right)$ is the size of the maximum path partition of $T \backslash E\left(T_{\omega}(v)\right)=T \backslash E\left(P_{0}\right)$, so $\alpha=\left(a_{0}, a_{1}, \ldots, a_{n}\right)$ is a path partition of $\vec{T}_{v}$, and $s_{\alpha}=s_{\omega}\left(v_{1}\right)$ by Corollary 2.3 and $f_{t}(T, v) \geq s_{\omega}\left(v_{1}\right)=s_{\omega}(T)$.

Definition 1.9. ([12]) Given a sequence $S$ of pebbling moves on $G$, the transition digraph obtained from $S$ is a directed multigraph denoted $T(G, S)$ that has $V(G)$ as its vertex set. Each move $s \in S$ along edge $u v$ (move off two pebbles from $u$ and add one on $v$ ) is represented by a directed edge $u v$.

The following lemma is useful in the following sections.

Lemma 1.10. ([12], No-Cycle Lemma) Let $S$ be a sequence of pebbling moves on $G$, reaching a distribution $D$. Then there exists a sequence $S^{*}$ of pebbling moves, thus reaching a distribution $D^{*}$ where

1. On each vertex $v, D^{*}(v) \geq D(v)$;

2. $T\left(G, S^{*}\right)$ does not contain any directed cycles.

\section{PRELIMINARIES}

We first introduce the path partition and the pebbling number of trees.

Definition 2.1. ([4]) Given a root $v$ of a tree $T$, then we can view $T$ as a directed graph $\overrightarrow{T_{v}}$ with edges directed toward $v . A$ path partition is a set of nonoverlapping directed paths in which the union is $\vec{T}_{v}$. A path partition is said to majorize another if the non-increasing sequence of the path size majorizes that of the other (that is $\left(a_{1}, a_{2}, \ldots, a_{r}\right)>\left(b_{1}, b_{2}, \ldots, b_{t}\right)$ if and only if $a_{i}>b_{i}$, where $\left.i=\min \left\{j: a_{j} \neq b_{j}\right\}\right)$. A path partition of a tree $\overrightarrow{T_{v}}$ is said to be maximum if it majorizes all other path partitions. Note that, in this paper, the sequence of the size of a path partition is always non-increasing. 
Note: By the definition of the maximum path partition, we can give a way to determine the size of the maximum path partition. First, we choose the longest directed path $P_{1}$ in $\overrightarrow{T_{v}}$, with length $a_{1}$. Then, we choose the longest directed path $P_{2}$ in $\vec{T}_{v} \backslash E\left(P_{1}\right)$, with length $a_{2}$, and so on. Moreover, it should be noted that the maximum path partition may not be unique, but the size of the maximum path partition must be unique.

Moews [4] found the $t$-pebbling number of trees by a path partition.

Theorem 2.2. ([4]) Let $T$ be a tree, $v \in V(T)$, and $\left(a_{1}, \ldots, a_{n}\right)$ be the size of the maximum path partition of $\vec{T}_{v}$. Then,

$$
\begin{gathered}
f_{t}(T, v)=t 2^{a_{1}}+\sum_{i=2}^{n} 2^{a_{i}}-n+1, \\
f_{t}(T)=\max _{v \in V(T)} f_{t}(T, v) .
\end{gathered}
$$

Corollary 2.3. Let $T$ be a tree, $v \in V(T)$, and $\alpha=\left(a_{1}, \ldots, a_{n}\right)$ be the size of a path partition of $\vec{T}_{v}, s_{\alpha}:=t 2^{a_{1}}+\sum_{i=2}^{n} 2^{a_{i}}-$ $n+1$. Then,

$$
f_{t}(T, v)=\max _{\alpha} s_{\alpha}
$$

Proof. Let $\alpha_{0}$ be the size of the maximum path partition of $\vec{T}_{v}$. Then, $f_{t}(T, v)=s_{\alpha_{0}} \leq \max _{\alpha} s_{\alpha}$.

Assume $P_{1}, P_{2}, \ldots, P_{n}$ is a path partition of $\vec{T}_{v}$, and the length of $P_{i}$ is $a_{i}$ for $1 \leq i \leq n$. Note that for each $P_{i}$ we should assume the two endpoints $v_{i}$ and $v_{i}^{\prime}$ satisfy $d\left(v_{i}, v\right)>d\left(v_{i}^{\prime}, v\right)$. We put $t 2^{a_{1}}-1$ pebbles on $v_{1}$ and $2^{a_{i}}-1$ pebbles on $v_{i}$ for $2 \leq i \leq n$; it is clear that $t$ pebbles cannot be moved to $v$ from this distribution. Thus, for each $\alpha, s_{\alpha}-1<f_{t}(T, v)$, so $s_{\alpha} \leq f_{t}(T, v)$ so $\max _{\alpha} s_{\alpha} \leq f_{t}(T, v)$.

Definition 2.4. Let $C$ be a generalized distribution on $G$, where $C(v)$ is an integer (may be negative) for all $v \in V(G)$. A pebbling move on $G$ consists of the removal of two pebbles from a vertex $v$ (with $C(v) \geq 2$ ) and the placement of one pebble on an adjacent vertex.

In the following, a distribution $D$ means that $D(v) \geq 0$, and a generalized distribution $C$ means $C(v)$ is an integer for all $v \in$ $V(G)$.

Definition 2.5. A pebbling move from $u$ to $v$ under a distribution $D$ is executable if $D(u) \geq 2$. A pebbling sequence $S$ is a finite set of pebbling moves, assuming $S=\left(S_{1}, \ldots, S_{k}\right)$, where $S_{i}$ is a pebbling move for $1 \leq i \leq k$, and the pebbling move $S_{i}$ is in front of $S_{j}$ if $1 \leq i<j \leq k$. We say the pebbling sequence $S$ executable, if $S_{i}$ is executable for $1 \leq i \leq k$.

Definition 2.6. Let $\omega$ be a nonnegative function on $V(G)$ and $C$ be a generalized distribution on $V(G)$. We say $C$ is $\omega$-solvable, if we can reach a distribution $C^{*}$ from $C$, by a sequence of pebbling moves so that $C^{*}(v) \geq \omega(v)$. In particular, if $\omega(v)=0$ for all $v \in V(G)$, then we say that $C$ is 0 -solvable.
Lemma 2.7. Let $D$ be a distribution on a graph $G$ and $\omega$ be a nonnegative function on $V(G), C:=D-\omega$. Then, $D$ is $\omega$-solvable if and only if $C$ is 0-solvable.

Proof. If $C$ is 0 -solvable, let $\delta$ be an executable pebbling sequence that reaches a distribution $D^{*}$ so that $D^{*}>0$ from $C$. It is then clear that $\delta$ is also an executable pebbling sequence that can reach a distribution $D^{\prime}$ so that $D^{\prime}=D^{*}+\omega>\omega$ from $D$. Thus $D$ is $\omega$-solvable.

On the other hand, if $D$ is $\omega$-solvable, by Lemma 1.10, there exists a pebbling sequence $S$ reaching a distribution $D^{*}$ with $D^{*}(v) \geq \omega(v)$, and $T(G, S)$ does not contain any direct cycle. We can thus give a sequence of the vertices of $G$, as $\left(v_{1}, v_{2}, \ldots, v_{n}\right)$, so that each directed edge $v_{i} v_{j}$ in $T(G, S)$ satisfies $i<j$. We can thus rearrange the sequence of pebbling moves $S$ along the order $\left(v_{1}, v_{2}, \ldots, v_{n}\right)$. It means we first choose all pebbling moves in $S$ that remove pebbles from $v_{1}$, choose all pebbling moves in $S$ that remove pebbles from $v_{2}$, and so on, and we denote this sequence of pebbling moves by $S^{\prime}$. We will show that $S^{\prime}$ is an executable pebbling sequence that reach $D^{*}-\omega$ from $C$.

In $S^{\prime}$, for each vertex $v \in V(G)$, the pebbling moves that move pebbles to $v$ are in front of the pebbling moves that remove pebbles from $v$. We may thus assume that, for each vertex $v_{i}$, we first move $\alpha_{i}$ pebbles from other vertices to $v_{i}$ and then remove $\beta_{i}$ pebbles from $v_{i}$.

We only need to show that, for each $v_{i} \in V(G)$, the sequence of pebbling moves that removes $\beta_{i}$ pebbles from $v_{i}$ in $S^{\prime}$, denoted by $\sigma_{i}$, is executable. We use induction on $i$. If $i=1$, and we can then get $D\left(v_{1}\right)-\beta_{1}=D^{*}\left(v_{1}\right) \geq \omega\left(v_{1}\right) \Rightarrow D\left(v_{1}\right)-\omega\left(v_{1}\right) \geq \beta_{1} \Rightarrow$ $C\left(v_{1}\right) \geq \beta_{1}$, and so $\sigma_{1}$ is executable.

Assume $\sigma_{h}$ is executable for $h<i$. By induction, the pebbling sequence that moves $\alpha_{i}$ pebbles to $v_{i}$ is executable. Moreover, we can get $D\left(v_{i}\right)+\alpha_{i}-\beta_{i}=D^{*}\left(v_{i}\right) \Rightarrow D\left(v_{i}\right)+\alpha_{i}-\omega\left(v_{i}\right)-\beta_{i}=$ $D^{*}\left(v_{i}\right)-\omega\left(v_{i}\right) \geq 0 \Rightarrow D\left(v_{i}\right)-\omega\left(v_{i}\right)+\alpha_{i} \geq \beta_{i} \Rightarrow C\left(v_{i}\right)+\alpha_{i} \geq \beta_{i}$. Thus $\sigma_{i}$ is executable.

So $S^{\prime}$ is an executable pebbling sequence that reaching $D^{*}-$ $\omega$ from $C$. Note that $D^{*}-\omega \geq 0$, and this completes the proof.

Definition 2.8. Let $D$ be a distribution on a tree $T$ and $\omega$ be a nonnegative function on $V(T) . C:=D-\omega$ is called the induced generalized distribution from $D$ and $\omega$ of $T$. Let $v$ be a leaf of $T$ and $u$ be its neighbor in $T$. The induced generalized distribution $C^{\prime}$ on $T \backslash v$ is given: if $C(v) \geq 0$, then $C^{\prime}(u)=C(u)+\lfloor C(v) / 2\rfloor$, and if $C(v)<0$, then $C^{\prime}(u)=C(u)+2 C(v)$, keeping $C^{\prime}(x)=C(x)$ unchanged for all $x \neq u$.

Lemma 2.9. Let $D$ be a distribution on a tree $T$ and $\omega$ be a nonnegative function on $V(T) . C:=D-\omega, v$ is a leaf of $T$, and $C^{\prime}$ is the induced generalized distribution from $D$ and $\omega$ of $T \backslash v$. Then, $C$ is 0 -solvable in $T$ if and only if $C^{\prime}$ is 0 -solvable in $T \backslash v$.

Proof. Firstly, we assume $C$ is 0 -solvable in $T$, and there is a pebbling sequence $\sigma$ reaching a distribution $C^{*}$ from $C$ with $C^{*}(x) \geq 0$ for each $x \in V(T)$.

Case 1.1. $C(v) \geq 0$. By Lemma 1.10, we may assume that no pebble has been moved from $u$ to $v$; at most, therefore, $\lfloor C(v) / 2\rfloor$ 
pebbles can be moved from $v$ to $u$. We may assume the first step of $\sigma$ is to move $\lfloor C(v) / 2\rfloor$ pebbles from $v$ to $u$, so the left steps makes $C^{\prime}$ solve 0 on $T \backslash v$.

Case 1.2. $C(v)<0$. By Lemma 1.10 , we may assume that no pebble has been moved from $v$ to $u$. So we may assume the last step of $\sigma$ is to move $-C(v)$ pebbles from $u$ to $v$, and so the steps before it makes $C^{\prime}$ solve 0 on $T \backslash v$.

Secondly, we assume $C^{\prime}$ is 0 -solvable in $T \backslash v$, and there is a pebbling sequence $\delta$ reaching a distribution $C^{*}$ from $C^{\prime}$ with $C^{*}(x) \geq 0$ for each $x \in V(T \backslash v)$.

Case 2.1. $C(v) \geq 0$. First, we move $\lfloor C(v) / 2\rfloor$ pebbles from $v$ to $u$, and the left steps are just $\delta$; this sequence makes $C$ solve 0 .

Case 2.2. $C(v)<0$. After the pebbling sequence $\delta$, we move $-C(v)$ pebbles from $u$ to $v$; this sequence makes $C$ solve 0 .

Notations: Assume $T^{*}$ is a subtree of $T$, then $T^{*}$ can be obtained from $T$ by deleting the leaves of the subtree of $T$ (the vertex with degree one) one by one. For each subtree $T^{*}$ of $T$, therefore, we can get an induced generalized distribution $C^{*}$. In particular, for each vertex $v \in V(T)$, let $T_{v}$ be a subtree containing $v$ and all of its neighbors. We use $C_{v}$ to denote the induced generalized distribution from $D$ and $\omega$ of $T_{v}$ and $\widehat{C}(v)$ to denote the induced generalized distribution of $\{v\}$.

Corollary 2.10. Let $D$ be a distribution on a tree $T, \omega$ be a nonnegative function on $V(T)$, and $\widehat{C}(v)$ be the induced generalized distribution from $D$ and $\omega$ of $\{v\}$. D is not $\omega$-solvable is equivalent to $\widehat{C}(v)<0$ for each $v \in V(T)$.

Proof. From Lemma 2.7 and Lemma 2.9, the result follows by induction.

Lemma 2.11. Let $D$ be a distribution on a tree $T$, which is not $\omega$ solvable with $|D|=\gamma_{\omega}(T)-1$. For each vertex $x \in V(T)$, which is not a leaf of $T$, there exists a vertex $y \in N(x)$, so that $C_{x}(y) \geq 0$.

Proof. If $C_{x}\left(x^{\prime}\right)<0$, for all $x^{\prime} \in N(x)$, assume $y, z \in N(x)$ with $C_{x}(z) \leq C_{x}(y)<0$. We delete all other vertices to the left $T_{1}=y x z$ and its induced generalized distribution $C_{1}$. Then, $C_{1}(y)=C_{x}(y), C_{1}(z)=C_{x}(z)$ and $\widehat{C}(x)=C_{1}(x)+2 C_{1}(y)+$ $2 C_{1}(z) \leq-1$ by Corollary 2.10. Note that $C_{1}(x)=D(x)-$ $w(x)+\sum_{x^{\prime} \in N(x), x^{\prime} \notin\{y, z\}} 2 C_{x}\left(x^{\prime}\right)$. Thus, $C_{1}(x)-D(x) \leq 0$ and $C_{1}(x)+2 C_{1}(z)-D(x) \leq 0$. Now, we remove $D(x)$ pebbles from $x$ and put $D(x)+1$ pebbles on $y$ to get a new distribution $D^{\prime}$ with $\left|D^{\prime}\right|=|D|+1$. The induced generalized distribution from $D^{\prime}$ and $\omega$ of $\{y\}$ is denoted by $\widehat{C}^{\prime}(y)$. Then, $\widehat{C}^{\prime}(y)=\left(C_{1}(y)+D(x)+1\right)+$ $2\left(C_{1}(x)+2 C_{1}(z)-D(x)\right)=\left(C_{1}(x)+2 C_{1}(y)+2 C_{1}(z)\right)+\left(C_{1}(z)-\right.$ $\left.C_{1}(y)\right)+C_{1}(z)+\left(C_{1}(x)-D(x)\right)+1 \leq-1+0-1+0+1=-1$, and so $D^{\prime}$ is not $\omega$-solvable by Corollary 2.10 , a contradiction to $\left|D^{\prime}\right|=\gamma_{\omega}(T)$, and we are done.

Theorem 2.12. Let $\omega$ be a nonnegative function on $V(T)$ and $D$ be a distribution that is not $\omega$-solvable with $|D|=\gamma_{\omega}(T)-1$. All pebbles are then distributed on the leaves of $T$.

Proof. If $D(x)>0$ for some vertex $x \in V(T)$, which is not a leaf, then $N(x)$ has at least two vertices. By Lemma 2.11, there exists a vertex $y \in N(x)$ with $C_{x}(y) \geq 0$. We first show that there exists a vertex $z \in N(x)$ with $C_{x}(z)<0$.

If not, that means for all $v \in N(x), C_{x}(v) \geq 0$. Note that $D(x)>0$, and thus $\widehat{C}(x)=D(x)+\sum_{v \in N(x)}\left\lfloor C_{x}(v) / 2\right\rfloor>0$. By Corollary 2.10, $D$ is $\omega$-solvable, a contradiction. Thus, there exists a vertex $z \in N(x)$ with $C_{x}(z)<0$.

Let $T_{1}=y x z$ be the subtree of $T$, with induced generalized distribution $C_{1}$. Then, $C_{1}(z)=C_{x}(z), C_{1}(y)=C_{x}(y)$, and $\widehat{C}(x)=C_{1}(x)+\left\lfloor C_{1}(y) / 2\right\rfloor+2 C_{1}(z)<0$.

Now, consider the new distribution $D^{*}$, with $D^{*}(y)=D(y)+$ $D(x)+1, D^{*}(x)=0$, and $D^{*}(v)=D(v) ;\left|D^{*}\right|=\gamma_{\omega}(T)$. The induced generalized distribution from $D^{*}$ and $\omega$ of $\{x\}$ is given by $\widehat{C^{*}}(x)=\left(C_{1}(x)-D(x)\right)+\left\lfloor\left(C_{1}(y)+D(x)+1\right) / 2\right\rfloor+2 C_{1}(z)$. If $D(x)=1$, then $\widehat{C^{*}}(x)=C_{1}(x)+\left\lfloor C_{1}(y) / 2\right\rfloor+2 C_{1}(z)=$ $\widehat{C}(x)<0$;

If $D(x) \geq 2$, then $\widehat{C^{*}}(x) \leq C_{1}(x)-D(x)+\left\lfloor C_{1}(y) / 2\right\rfloor+D(x) / 2+$ $1+2 C_{1}(z)=\widehat{C}(x)+1-\bar{D}(x) / 2 \leq \widehat{C}(x)<0$.

By Corollary $2.10, D^{*}$ is not $\omega$-solvable, a contradiction to $\left|D^{*}\right|=\gamma_{\omega}(T)$. This completes the proof.

From Theorem 2.12, for a given integer $p$ with $p<\gamma_{\omega}(T)$, there must exist a distribution $D$, which is not $\omega$-solvable with $|D|=p$, and all pebbles are distributed on the leaves of $T$.

\section{THE GENERALIZATION OF THE COVER PEBBLING NUMBER ON TREES}

Assume that $s_{\omega}\left(v_{0}\right)=s_{\omega}(T)$ for some $v_{0} \in V(T)$; it should be noted that $\vec{T} \backslash E\left(T_{\omega}\left(v_{0}\right)\right)$ is a directed graph. We define $d_{\omega}(u, l)$ to be the length of the maximal path containing $u$ in all maximum path partitions of $\vec{T} \backslash E\left(T_{\omega}\left(v_{0}\right)\right)$. If $\omega$ is clear, then we use $d(u, l)$ for short (note that $d(u, l)$ maybe 0 ). Let $P_{\alpha}$ be a maximal path partition of $\vec{T} \backslash E\left(T_{\omega}\left(v_{0}\right)\right)$; then, $d_{\omega}(u, l)=\max _{P_{\alpha}}\{|P|: u \in$ $\left.P, P \in P_{\alpha}\right\}$.

Lemma 3.1. Assume that $s_{\omega}\left(v_{0}\right)=s_{\omega}(T)$ for some $v_{0} \in V(T)$; then for each vertex $u \in V(T)$ and $d\left(u, v_{0}\right) \geq d(u, l)$.

Proof. Assume $u, v \in V(T)$. There is exactly one subpath of $T$ with endpoints $u$ and $v$, and we denote this path by $P_{u v}$. We thus have $P_{u v}=P_{v u}$.

If $|W|=1$, we may assume that $\omega(v)=t$, and $\omega(u)=$ 0 for $u \neq v$. By the proof of Theorem 1.8, we know that $f_{t}(T, v)=s_{\omega}\left(v_{0}\right)$. Let $\left(a_{1}, a_{2}, \ldots, a_{n}\right)$ be the size of the maximum path partition of $\vec{T}_{v}$. Then $d\left(v, v_{0}\right)=\max _{u \in V(T)} d(v, u)=a_{1}$. Assume $P_{1}$ is the maximal path containing $u$ in $\vec{T}_{v} \backslash P_{v_{0}, v}$, and $P_{1} \cap P_{v_{0} v}=v_{1}$. The length of $P_{v_{0} v}\left(P_{1}\right)$ is thus $a_{1}(d(u, l))$ and $d\left(v_{1}, v_{0}\right) \leq d\left(u, v_{0}\right)$. If $d\left(u, v_{0}\right)<d(u, l)$, then $d\left(v_{1}, v_{0}\right)<$ $d(u, l)$, and we get a path $P_{1} \cup P_{v_{1} v}$ with length $a_{1}-d\left(v_{1}, v_{0}\right)+$ $d(u, l)>a_{1}$, a contradiction to the maximum of $a_{1}$, and thus $d\left(u, v_{0}\right) \geq d(u, l)$.

If $|W| \geq 2$, we only need to show it while $u \in V\left(T_{\omega}\left(v_{0}\right)\right)$.

If $d\left(u, v_{0}\right)<d(u, l)$ for some $u \in V\left(T_{\omega}\left(v_{0}\right)\right)$, there exists a leaf $v_{1}$ in $\vec{T} \backslash E\left(T_{\omega}\left(v_{0}\right)\right)$ so that $d(u, l)=d\left(u, v_{1}\right)$, and we will show that $s_{\omega}\left(v_{1}\right)>s_{\omega}\left(v_{0}\right)$. 
Let $T C(v)$ be the component of $T \backslash u$ containing the vertex $v$. We thus have $T C\left(v_{1}\right) \cap W=\emptyset$.

Case 1. $T C\left(v_{0}\right) \cap W \neq \emptyset$.

Assume $w_{1} \in T C\left(v_{0}\right) \cap W$, then $d\left(w_{1}, v_{1}\right) \geq d\left(u, v_{1}\right)+1$ and

$$
d\left(w_{1}, v_{1}\right)-d\left(w_{1}, v_{0}\right) \geq d\left(u, v_{1}\right)-d\left(u, v_{0}\right)+2 \geq 3 .
$$

Note that $\vec{T} \backslash E\left(T_{\omega}\left(v_{0}\right) \cup P_{v_{1} u}\right) \subseteq \vec{T} \backslash E\left(T_{\omega}\left(v_{1}\right)\right)$. So

$$
\begin{aligned}
& s_{\omega}\left(v_{1}\right)-s_{\omega}\left(v_{0}\right) \\
& \geq \sum_{x \in W} \omega(x)\left(2^{d\left(x, v_{1}\right)}-2^{d\left(x, v_{0}\right)}\right)-2^{d\left(u, v_{1}\right)} \\
& \geq \omega\left(w_{1}\right)\left(2^{d\left(w_{1}, v_{1}\right)}-2^{d\left(w_{1}, v_{0}\right)}\right)-2^{d\left(u, v_{1}\right)} \\
& \geq 2^{d\left(w_{1}, v_{1}\right)}-2^{d\left(w_{1}, v_{0}\right)}-2^{d\left(u, v_{1}\right)} \\
& \geq 2^{d\left(w_{1}, v_{1}\right)}-\frac{2^{d\left(w_{1}, v_{1}\right)}}{8}-\frac{2^{d\left(w_{1}, v_{1}\right)}}{2} \\
& =\frac{3 \cdot 2^{d\left(w_{1}, v_{1}\right)}}{8}>0 .
\end{aligned}
$$

Hence, $s_{\omega}\left(v_{1}\right)>s_{\omega}\left(v_{0}\right)$, which is a contradiction to $s_{\omega}\left(v_{0}\right)=$ $s_{\omega}(T)$.

Case 2. $T C\left(v_{0}\right) \cap W=\emptyset$.

Let $\tau_{\omega}(v)=\sum_{x \in W} \omega(x) 2^{d(x, v)}$. If so, then $\tau_{\omega}\left(v_{0}\right)=$ $2^{d\left(u, v_{0}\right)} \tau_{\omega}(u)$, and $\tau_{\omega}\left(v_{1}\right)=2^{d\left(u, v_{1}\right)} \tau_{\omega}(u)$. For $|W| \geq 2, \tau_{\omega}(u) \geq$ $2^{0}+2^{1}=3$.

Note that $\vec{T} \backslash E\left(T_{\omega}\left(v_{0}\right) \cup P_{v_{1} u}\right) \subseteq \vec{T} \backslash E\left(T_{\omega}\left(v_{1}\right)\right)$. So

$$
\begin{aligned}
& s_{\omega}\left(v_{1}\right)-s_{\omega}\left(v_{0}\right) \\
& \geq 2^{d\left(u, v_{1}\right)} \tau_{\omega}(u)-2^{d\left(u, v_{0}\right)} \tau_{\omega}(u)-2^{d\left(u, v_{1}\right)} \\
& =\tau_{\omega}(u)\left(2^{d\left(u, v_{1}\right)}-2^{d\left(u, v_{0}\right)}\right)-2^{d\left(u, v_{1}\right)} \\
& \geq 3\left(2^{d\left(u, v_{1}\right)}-2^{d\left(u, v_{0}\right)}\right)-2^{d\left(u, v_{1}\right)} \\
& \geq 3\left(2^{d\left(u, v_{1}\right)}-\frac{2^{d\left(u, v_{1}\right)}}{2}\right)-2^{d\left(u, v_{1}\right)} \\
& =\frac{2^{d\left(u, v_{1}\right)}}{2}>0 .
\end{aligned}
$$

Hence, $s_{\omega}\left(v_{1}\right)>s_{\omega}\left(v_{0}\right)$, which is a contradiction to $s_{\omega}\left(v_{0}\right)=$ $s_{\omega}(T)$, and this completes the proof.

Corollary 3.2. Let $\omega$ be a nonnegative function in $V(T)$, for some $v \in W$, and $\omega^{\prime}$ be a nonnegative function satisfying $\omega^{\prime}(v)=\omega(v)-1, \omega^{\prime}(u)=\omega(u)$ for other vertices in $T$. If so, then

$$
s_{\omega}(T) \geq s_{\omega^{\prime}}(T)+2^{d_{\omega}(v, l)} .
$$

Proof. Assume that there exist $v_{1}$ and $v_{2}$, so that $s_{\omega}\left(v_{1}\right)=s_{\omega}(T)$ and $s_{\omega^{\prime}}\left(v_{2}\right)=s_{\omega^{\prime}}(T)$.

By the definition of $s_{\omega}(v)$, if $\omega(v) \geq 2$, then $d_{\omega}(v, l)=d_{\omega^{\prime}}(v, l)$, we have

$$
\begin{aligned}
s_{\omega}(T)=s_{\omega}\left(v_{1}\right) & \geq s_{\omega}\left(v_{2}\right) \\
& =s_{\omega^{\prime}}\left(v_{2}\right)+2^{d\left(v, v_{2}\right)} \\
& \geq s_{\omega^{\prime}}\left(v_{2}\right)+2^{d_{\omega^{\prime}}(v, l)} \quad(\text { by Lemma 3.1) } \\
& =s_{\omega^{\prime}}(T)+2^{d_{\omega}(v, l)} .
\end{aligned}
$$

If $\omega(v)=1$, the difference between $\vec{T} \backslash T_{\omega}\left(v_{1}\right)$ and $\vec{T} \backslash T_{\omega^{\prime}}\left(v_{2}\right)$ is just the length of the maximal path containing $v$, we have

$$
\begin{aligned}
s_{\omega}(T)=s_{\omega}\left(v_{1}\right) & \geq s_{\omega}\left(v_{2}\right) \\
& =s_{\omega^{\prime}}\left(v_{2}\right)+2^{d\left(v, v_{2}\right)}+2^{d_{\omega}(v, l)}-2^{d_{\omega^{\prime}}(v, l)} \\
& \geq s_{\omega^{\prime}}\left(v_{2}\right)+2^{d_{\omega}(v, l)} \quad(\text { by Lemma 3.1) } \\
& =s_{\omega^{\prime}}(T)+2^{d_{\omega}(v, l)} .
\end{aligned}
$$

\section{The proof of Theorem 1.7:}

The lower bound holds clearly, as we put $2^{a_{i}}-1$ pebbles on the leaf of each path for $1 \leq i \leq n$ (no pebble can then be moved to $\left.T_{\omega}(v)\right)$, and $\sum_{u \in S} w(u) 2^{d(u, v)}-1$ pebbles on $v$, obviously it is not $\omega$-solvable.

For the upper bound, it holds if $|\omega|=1$ or $|W|=1$ by the proof of Theorem 1.8. It also holds for $|T| \leq 2$ by Theorem 2.2 and Theorem 1.4. We may thus assume that $|\omega| \geq 2,|W| \geq 2$, and $|T| \geq 3$.

If the result is false for some $T$ and $\omega$, then we choose one counterexample $T$ and its weight $\omega$ so that $|T|$ and $|\omega|$ are both minimal. It means the upper bound holds for $T^{\prime}$ and its weight $\omega^{\prime}$ if $\left|T^{\prime}\right|<|T|$ or $\left|\omega^{\prime}\right|<|\omega|$.

Let $D$ be a distribution on $T$, which is not $\omega$-solvable with size $s_{\omega}(T)$. By Theorem 2.12, we may assume that all pebbles are distributed on the leaves of $T$.

Assume $s_{\omega}\left(v_{0}\right)=s_{\omega}(T)$. There exists $x \in W \backslash v_{0}$ satisfying $d_{T_{\omega}\left(v_{0}\right)}(x)=1$. If $d_{T}(x) \neq 1$, we can get $d(x, l)>0$, and there exists a nonempty component in $T \backslash E\left(T_{\omega}\left(v_{0}\right)\right)$, which is connected with $x$. Say $T_{1}$ and $b_{1} \geq b_{2} \geq \ldots \geq b_{m}$ is the size of the maximum path partition of $T_{1}$.

Case 1. $D\left(T_{1}\right)$ cannot move a pebble to $x \cdot\left|D\left(T_{1}\right)\right| \leq$ $\sum_{i=1}^{m} 2^{b_{i}}-m$, and we consider $D$ on $T \backslash T_{1},\left|D\left(T \backslash T_{1}\right)\right| \geq$ $s_{\omega}(T)-D\left(T_{1}\right) \geq s_{\omega}\left(T \backslash T_{1}\right)$, and $D\left(T \backslash T_{1}\right)$ is not $\omega$-solvable, a contradiction to the minimum of $|T|$.

Case 2. $D\left(T_{1}\right)$ can move one pebble to $x$. It costs us at most $2^{b_{1}}=2^{d_{\omega}(x, l)}$ pebbles on $T_{1}$. The left pebbles on $T$ is not $\omega^{\prime}$ solvable $\left(\omega^{\prime}\right.$ satisfies $\omega^{\prime}(x)=\omega(x)-1$, and it is unchanged for other vertices in $T$ ). From the minimum of $|\omega|$ and Corollary 3.2, we thus have $|D|<s_{\omega^{\prime}}(T)+2^{d_{\omega}(x, l)} \leq s_{\omega}(T)$, a contradiction to $|D|=s_{\omega}(T)$.

We may therefore assume $d_{T}(x)=1$.

We claim that $D(x)=0$. Otherwise, let $\omega^{\prime}$ satisfy $\omega^{\prime}(x)=$ $\omega(x)-1$ and $\omega^{\prime}(v)=\omega(v)$ for $v \neq x$. Regardless of one pebble being on $x$, we know that $|D|-1$ other pebbles cannot solve $\omega^{\prime}$. From the minimum of $|\omega|$, we have $|D|-1 \leq s_{\omega^{\prime}}(T)-1$. By Corollary 3.2, $s_{\omega^{\prime}}(T)+1 \leq s_{\omega}(T)$, so $|D| \leq s_{\omega}(T)-1$, a contradiction to $|D|=s_{\omega}(T)$, so $D(x)=0$.

Assuming that $x^{\prime} \sim x$ in $T$, we then delete $x$. Let $C^{\prime}\left(x^{\prime}\right)=$ $C\left(x^{\prime}\right)+2 C(x)$ and $C^{\prime}(v)=C(v)$ otherwise. Note that all pebbles are distributed on the leaves of $T$, so $C^{\prime}(x)=D\left(x^{\prime}\right)-\omega\left(x^{\prime}\right)-$ $2(D(x)-\omega(x))=-\omega\left(x^{\prime}\right)-2 \omega(x)$. By Lemma 2.9, $D$ is not $\omega-$ solvable in $T$ is equivalent to $D$ is not $\omega^{\prime}$-solvable in $T \backslash x$, where $\omega^{\prime}\left(x^{\prime}\right)=\omega\left(x^{\prime}\right)+2 \omega(x)$ and $\omega^{\prime}(v)=\omega(v)$ for $v \neq x$. By the minimum of $|T|$, we have $|D| \leq s_{\omega^{\prime}}(T \backslash x)-1$, note that $x \neq v_{0}$, we have $s_{\omega^{\prime}}(T \backslash x)=s_{\omega}(T)$, a contradiction to $|D|=s_{\omega}(T)$. This completes the proof. 
Moreover, by Theorem 1.7, we can immediately get

Corollary 3.3. Let $T$ be a tree, and let $\omega$ be a nonnegative function on $V(T), W=\{v \in V(T): \omega(v)>0\}, L=\{v \in V(T): d(v)=1\}$, then if $L \subseteq W$,

$$
\gamma_{\omega}(T)=\max _{v \in V(T)} \sum_{u \in V(T)} \omega(u) 2^{d(u, v)} .
$$

Theorem 1.4 gives a sufficient condition of a nonnegative weight function $\omega$ on $V(G)$ for a graph $G$ so that the $\omega$-cover pebbling number of $G$ is

$$
\gamma_{\omega}(G)=\max _{v \in V(G)} \sum_{u \in V(G)} \omega(u) 2^{d(u, v)} .
$$

Corollary 3.3 gives a weaker sufficient condition of a nonnegative weight function $\omega$ on $V(T)$ for a tree $T$ so that the $\omega$-cover pebbling number of $T$ is

$$
\gamma_{w}(T)=\max _{v \in V(T)} \sum_{u \in V(T)} \omega(u) 2^{d(u, v)} .
$$

Here, we explore some problems.

Problem 3.4. Give a weaker sufficient condition of a nonnegative function $\omega$ on $V(G)$ for a graph $G$ so that the $\omega$-cover pebbling number of $G$ is

$$
\gamma_{\omega}(G)=\max _{v \in V(G)} \sum_{u \in V(G)} \omega(u) 2^{d(u, v)} .
$$

Problem 3.5. For a nonnegative function $\omega$, determine the $\omega$ cover pebbling number of more graphs, such as cycles, hypercubes, and so on.

\section{REFERENCES}

1. Chung FRK. Pebbling in hypercubes. SIAM J Discrete Math. (1989) 2:467-72. doi: 10.1137/0402041

2. Lewis T, Cusack CA, Dion L. The complexity of pebbling reachability and solvability in planar and outerplanar graphs. Discrete Appl Math. (2014) 172:62-74. doi: 10.1016/j.dam.2014.03.008

3. Milans K, Clark B. The complexity of graph pebbling. SIAM J Discrete Math. (2006) 20:769-98. doi: 10.1137/050636218

4. Moews D. Pebbling graphs. J Combin Theory Ser B. (1992) 55:244-52. doi: 10.1016/0095-8956(92)90043-W

5. Pachter L, Snevily HS, Voxman B. On pebbling graphs. Congr Numer. (1995) 107:65-80. doi: 10.1016/S0166-218X(97)00073-5

6. Snevily HS, Foster JA. The 2-pebbling property and conjecture of Graham's. Graphs Combin. (2000) 16:231-44. doi: 10.1007/PL00021179

7. Xia ZJ, Hong ZM. Generalization of the Cover Pebbling Number on Trees (2019). Available online at: http://export.arxiv.org/pdf/1903.04867.

8. Hurlbert GH. General graph pebbling. Discrete Appl Math. (2013) 16:122131. doi: 10.1016/j.dam.2012.03.010

9. Liu J-B, Wang S, Wang C, Hayat S. Further results on computation of topological indices of certain networks. IET
We also give a conjecture which is similar to Graham's Conjecture.

Conjecture 3.6. Let $\omega_{1}$ be a nonnegative function on $G$ and $\omega_{2}$ be a nonnegative function on $H$. The function $\omega$ on $G \times H$ is given by $\omega((g, h))=\omega_{1}(g) \omega_{2}(h)$, where $g \in V(G)$ and $h \in V(H)$, then $\gamma_{\omega}(G \times H) \leq \gamma_{\omega_{1}}(G) \gamma_{\omega_{2}}(H)$.

\section{DATA AVAILABILITY STATEMENT}

The original contributions presented in the study are included in the article/supplementary materials, further inquiries can be directed to the corresponding authors.

\section{AUTHOR CONTRIBUTIONS}

Z-JX provided this topic and wrote the paper. Z-JX and $\mathrm{Z}-\mathrm{MH}$ solved the problem. Z-MH reviewed and edited the manuscript. All authors contributed to the article and approved the submitted version.

\section{FUNDING}

This research was supported by Key Projects in Natural Science Research of Anhui Provincial Department of Education (No. KJ2018A0438) to (Z-JX) and by NSFC (No. 11601002) to $(\mathrm{Z}-\mathrm{MH})$.

\section{ACKNOWLEDGMENTS}

This manuscript has been released as a pre-print at http:// export.arxiv.org/pdf/1903.04867 (Z-JX and Z-MH). The authors are grateful for the many useful comments provided by the referees.

Control Theory Appl. (2017) 11:2065-71. doi: 10.1049/iet-cta.20 16.1237

10. Liu J-B, Wang WR, Zhang YM, Pan XF. On degree resistance distance of cacti. Discrete Appl Math. (2016) 203:217-25. doi: 10.1016/j.dam.2015. 09.006

11. Sjostrand J. The cover pebbling theorem. Electron J Combin. (2005) 12:5. doi: $10.37236 / 1989$

12. Crull B, Cundif T, Feltman P, Hurlbert G, Pudwell L, Szaniszlo Z, et al. The cover pebbling number of graphs. Discrete Math. (2005) 296:15-23. doi: 10.1016/j.disc.2005.03.009

Conflict of Interest: The authors declare that the research was conducted in the absence of any commercial or financial relationships that could be construed as a potential conflict of interest.

Copyright (C) $2020 \mathrm{Xia}$ and Hong. This is an open-access article distributed under the terms of the Creative Commons Attribution License (CC BY). The use, distribution or reproduction in other forums is permitted, provided the original author(s) and the copyright owner(s) are credited and that the original publication in this journal is cited, in accordance with accepted academic practice. No use, distribution or reproduction is permitted which does not comply with these terms. 